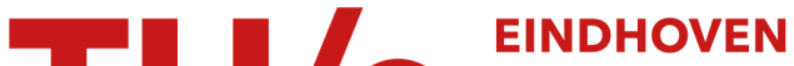 \\ UNIVERSITY OF \\ TECHNOLOGY
}

\section{Generalised soundness of workflow nets is decidable}

Citation for published version (APA):

Voorhoeve, M., Hee, van, K. M., \& Sidorova, N. (2003). Generalised soundness of workflow nets is decidable. (Computer science reports; Vol. 0315). Technische Universiteit Eindhoven.

Document status and date:

Published: 01/01/2003

\section{Document Version:}

Publisher's PDF, also known as Version of Record (includes final page, issue and volume numbers)

\section{Please check the document version of this publication:}

- A submitted manuscript is the version of the article upon submission and before peer-review. There can be important differences between the submitted version and the official published version of record. People interested in the research are advised to contact the author for the final version of the publication, or visit the $\mathrm{DOI}$ to the publisher's website.

- The final author version and the galley proof are versions of the publication after peer review.

- The final published version features the final layout of the paper including the volume, issue and page numbers.

Link to publication

\section{General rights}

Copyright and moral rights for the publications made accessible in the public portal are retained by the authors and/or other copyright owners and it is a condition of accessing publications that users recognise and abide by the legal requirements associated with these rights.

- Users may download and print one copy of any publication from the public portal for the purpose of private study or research.

- You may not further distribute the material or use it for any profit-making activity or commercial gain

- You may freely distribute the URL identifying the publication in the public portal.

If the publication is distributed under the terms of Article $25 \mathrm{fa}$ of the Dutch Copyright Act, indicated by the "Taverne" license above, please follow below link for the End User Agreement:

www.tue.nl/taverne

Take down policy

If you believe that this document breaches copyright please contact us at:

openaccess@tue.nl

providing details and we will investigate your claim. 


\title{
Generalised Soundness of Workflow Nets is Decidable
}

\author{
Kees van Hee, Natalia Sidorova, and Marc Voorhoeve \\ Department of Mathematics and Computer Science \\ Eindhoven University of Technology \\ P.O. Box 513, 5600 MB Eindhoven, The Netherlands \\ k.m.v.hee@tue.nl, n.sidorova@tue.nl, m.voorhoeve@tue.nl
}

\begin{abstract}
We investigate the decidability of the problem of generalised soundness for Workflow nets: "Every marking reachable from an initial marking with $k$ tokens on the initial place terminates properly, i.e. it can reach a marking with $k$ tokens on the final place, for an arbitrary natural number $k$ ". We start with considering simple correctness criteria for Workflow nets and reduce them to the check of structural properties formulated in terms of traps and siphons, which can be easily checked. We call the nets that possess those properties Batch Workflow nets (BWF-nets). We show that every WF-net is either not sound or it can be transformed to a BWF-net with the same behaviour. Then we use algebraic methods to prove that generalized soundness is decidable for BWF-nets and give a decision procedure.

Keywords: Petri nets; workflows; verification; soundness, decidability.
\end{abstract}

\section{Introduction}

Petri nets are widely used for the modelling and verification of workflows. In [1], the class of Workflow (Petri) nets (WF-nets) was defined. A Petri net is a WF-net iff it satisfies certain structural properties, namely it possesses one source place (initial place) and one sink place (final place) and all other nodes lie on paths from the source to the sink place. The main correctness criterion introduced there was soundness. The requirements a sound workflow net should meet are (1) the reachability of a marking with a single token on the final place from any marking reachable from the marking with one token on the initial place, (2) the uniqueness of the marking containing a token on the final place, and (3) the absence of dead transitions for the net with a single token on the initial place. Soundness for WF-nets is decidable, and the decision procedure has been implemented e.g. in the WOFLAN tool [8].

In [4] we showed that the notion of soundness from [1] is not compositional, and moreover, it does not allow for handling of multiple cases in the WF-net. We introduced there a generalized soundness notion that amounts to proper termination of all markings obtained from markings with multiple tokens on the initial place, which corresponds to the processing of batches of cases in the WF-net. With proper termination for marking $m$ obtained from a marking with $k$ tokens on the initial place, we mean that there exists a firing sequence leading from $m$ to the marking with $k$ tokens on the final place. We proved that generalised soundness is compositional. The original soundness notion from [1] corresponds to 1-soundness in our case. Deciding generalised soundness is harder than deciding 1-soundness, since the straightforward approach involves an infinite number of checks of 
proper termination. We did not solve the problem of soundness in [4] but defined a class of nets (ST-nets) that are sound by construction. In this paper we prove that the problem of (generalised) soundness is decidable for arbitrary WF-nets and describe a decision procedure for it.

We start with considering simple behavioural correctness criteria for WF-nets: non-redundancy and non-persistency. Non-redundancy means that every place can be marked and every transition can fire, provided that the initial place contains enough tokens, while nonpersistency means that all places (except for the final one) can become empty again, lest some garbage would be left after the processing of the case is finished. We show that the WF-nets meet non-redundancy and non-persistency requirements iff they satisfy a simple structural characterisation: all proper siphons of these nets contain the initial place and all proper traps contain the final place. We call this class of WF-nets Batch Workflow nets (BWF-nets). We show that every WF-net is either not sound (in case it contains persistent places) or it can be transformed to a BWF-net with the same behaviour (by removing redundant places and transitions).

In the second half of the paper we consider the problem of (generalised) soundness for BWF-nets and prove that this problem is decidable. The decidability proof is based on two ideas. First, we extend the set $\mathcal{R}$ of markings reachable from the initial markings upto a set $\mathcal{G}$ that has a 'regular' algebraic structure and show that the notion of soundness can be equally defined by requiring proper termination of all markings of this extended set $\mathcal{G}$. And second, we use the regularity of the structure of this set to show that it is enough to check proper termination for the markings of a finite subset $\Gamma$ of $\mathcal{G}$ to prove the proper termination of all markings from $\mathcal{G}$. Thus, the infinite set of markings in the initial formulation of the problem of generalised soundness can be reduced to a finite set due to the clean algebraic model of Petri nets.

The rest of the paper is organised as follows. In Section 2, we sketch the basic definitions related to Petri nets and Workflow nets. In Section 3 we introduce the notion of Batch Workflow Nets. In Section 4 we prove that the problem of generalised soundness is decidable. In Section 5 we illustrate the decision procedure for soundness on a concrete example. We conclude in Section 6 with discussion of the obtained results and directions for future work.

\section{Preliminaries}

$\mathbb{N}$ denotes the set of natural numbers, $\mathbb{Z}$ the set of integers and $\mathbb{Q}$ the set of rational numbers. $\mathbb{Q}^{+}$stands for the set of non-negative rational numbers.

Let $P$ be a set. A bag (multiset) $m$ over $P$ is a mapping $m: P \rightarrow \mathbb{N}$. The set of all bags over $P$ is $\mathbb{N}^{P}$. We use + and - for the sum and the difference of two bags and $=,<,>, \leq, \geq$ for comparisons of bags, which are defined in a standard way. We overload the set notation, writing $\emptyset$ for the empty bag and $\in$ for the element inclusion. We write $m=2[p]+[q]$ for a bag $m$ with $m(p)=2, m(q)=1$, and $m(x)=0$ for all $x \notin\{p, q\}$. For a sum over the 
elements of a bag $m$ we write $\sum_{p \in m} f(p)$ (assuming that every $p$ appears in the sum $m(p)$ times) rather than $\sum_{p \in m} m(p) \cdot f(p)$.

For (finite) sequences of elements over a set $T$ we use the following notation: The empty sequence is denoted with $\epsilon$; a non-empty sequence can be given by listing its elements between angle brackets. The Parikh vector $\vec{\sigma}: T \rightarrow \mathbb{N}$ of a sequence $\sigma$ maps every element $t \in T$ to the number of occurrences of $t$ in $\sigma$.

Transition Systems A transition system is a tuple $E=\langle S$, Act, $T\rangle$ where $S$ is a set of states, Act is a finite set of action names and $T \subseteq S \times$ Act $\times S$ is a transition relation. A process is a pair $\left\langle E, s_{0}\right\rangle$ where $E$ is a transition system and $s_{0} \in S$ an initial state. We denote $\left(s_{1}, a, s_{2}\right)$ from $T$ as $s_{1} \stackrel{a}{\longrightarrow} s_{2}$, and we say that $a$ leads from $s_{1}$ to $s_{2}$. For a sequence of transitions $\sigma=\left\langle t_{1}, \ldots, t_{n}\right\rangle$ we write $s_{1} \stackrel{\sigma}{\longrightarrow} s_{2}$ when $s_{1}=s^{0} \stackrel{t_{1}}{\longrightarrow} s^{1} \stackrel{t_{2}}{\longrightarrow} \ldots \stackrel{t_{n}}{\longrightarrow} s^{n}=s_{2}$, and $s_{1} \stackrel{\sigma}{\longrightarrow}$ when $s_{1} \stackrel{\sigma}{\longrightarrow} s_{2}$ for some $s_{2}$. In this case we say that $\sigma$ is a trace of $E$. Finally, $s_{1} \stackrel{*}{\longrightarrow} s_{2}$ means that there exists a sequence of transitions $\sigma \in T^{*}$ such that $s_{1} \stackrel{\sigma}{\longrightarrow} s_{2}$.

Petri nets A Petri net is a tuple $N=\left\langle P, T, F^{+}, F^{-}\right\rangle$, where:

- $P$ and $T$ are two disjoint non-empty finite sets of places and transitions respectively, the set $P \cup T$ are the nodes of $N$;

$-F^{+}$and $F^{-}$are mappings $(P \times T) \rightarrow \mathbb{N}$ that are flow functions from transitions to places and from places to transitions respectively.

$F=F^{+}-F^{-}$is the incidence matrix of net $N$.

We present nets with the usual graphical notation.

Markings are states (configurations) of a net. Depending on the context, we interprete a marking $m$ of $N$ either as a bag over $P$ (in Section 3 ) or as a vector from $P \rightarrow \mathbb{N}$ (in Sections 4 and 5)). We denote the set of all markings reachable in net $N$ from marking $m$ as $\mathcal{R}(m)$. The set of markings from which marking $m$ is reachable is denoted as $\mathcal{S}(m)$.

Given a transition $t \in T$, the preset ${ }^{\bullet} t$ and the postset $t^{\bullet}$ of $t$ are the bags of places where every $p \in P$ occurs in ${ }^{\bullet} t F^{-}(p, t)$ times and in $t^{\bullet} F^{+}(p, t)$ times. Analogously we write ${ }^{\bullet} p, p^{\bullet}$ for pre- and postsets of places. We overload this notation further and apply preset and postset operations to a set $B$ of places: ${ }^{\bullet} B=\left\{t \mid \exists p \in B: t \in{ }^{\bullet} p\right\}$ and $B^{\bullet}=\left\{t \mid \exists p \in B: t \in p^{\bullet}\right\}$. Note that ${ }^{\bullet} B$ and $B^{\bullet}$ are not bags but sets. We will say that node $n$ is a source node iff $n=\emptyset$ and $n$ is a sink node iff $n^{\bullet}=\emptyset$. A path of a net is a sequence $\left\langle x_{0}, \ldots, x_{n}\right\rangle$ of nodes such that $\forall i: 1 \leq i \leq n: x_{i-1} \in{ }^{\bullet} x_{i}$.

A transition $t \in T$ is enabled in marking $m$ iff $\bullet \leq m$. An enabled transition $t$ may fire. This results in a new marking $m^{\prime}$ defined by $m^{\prime} \stackrel{\text { def }}{=} m-\bullet t+t^{\bullet}$. We interpret a Petri net $N$ as a transition system/process where markings play the role of states, firings of the enabled transitions define the transition relation and the initial marking corresponds to the initial state. The notion of reachability for Petri nets is inherited from the transition systems. For a firing sequence $\sigma$ in a net $N$, we define $\bullet^{\circ}$ and $\sigma^{\bullet}$ respectively as $\sum_{t \in \sigma}{ }^{\bullet} t$ and $\sum_{t \in \sigma} t^{\bullet}$, which are the sums of all tokens consumed/produced during the firings of $\sigma$. So $m \stackrel{\sigma}{\longrightarrow}\left(m+\sigma^{\bullet}-\bullet^{\bullet}\right)$. We will use the well-known Marking Equation Lemma: 
Lemma 1 (Marking Equation). Given a finite firing sequence $\sigma$ of a net $N: m \stackrel{\sigma}{\longrightarrow} m^{\prime}$, the following equation holds: $m^{\prime}=m+F^{+} \cdot \vec{\sigma}-F^{-} \cdot \vec{\sigma}$, or in other words, $m^{\prime}=m+F \cdot \vec{\sigma}$.

Note that the reverse is not true: not every marking $m^{\prime}$ that is representable as a sum $m+F \cdot v$ for some $v \in \mathbb{N}^{T}$ is reachable from the marking $m$.

Traps and Siphons (see [2]) A set $R$ of places is a trap if $R^{\bullet} \subseteq \bullet$. The trap is a proper trap iff it is not empty. A set $R$ of places is a siphon if $R \subseteq R^{\bullet}$. The siphon is a proper siphon iff it is not empty. Important properties of traps and siphons are that marked traps remain marked and unmarked siphons remain unmarked whatever transition firings would happen. As follows from the definition, traps and siphons are dual by their nature.

Place Invariants (see [5]) A place invariant is a row vector $I: P \rightarrow \mathbb{Q}$ such that $I \cdot F=0$. When talking about invariants, we consider markings as vectors. We will say that markings $m_{1}$ and $m_{2}$ agree on a place invariant $I$ if $I \cdot m_{1}=I \cdot m_{2}$ (see [3]).

Lemma 2. Two markings $m_{1}, m_{2}$ agree on all place invariants iff the equation $m_{1}+F \cdot x=$ $m_{2}$ has some rational-valued solution for $x$.

The main property of place invariants is thus that any two markings $m_{1}, m_{2}$ such that $m_{1} \stackrel{*}{\longrightarrow} m_{2}$ agree on all place invariants. The check whether the two markings $m_{1}, m_{2}$ agree on all place invariants can be done by a simple check whether $\mathcal{I} \cdot m_{1}=\mathcal{I} \cdot m_{2}$, where $\mathcal{I}$ is a matrix that consists of basis place invariants as rows.

Workflow Petri nets In this paper we primarily focus upon the Workflow Petri nets (WF-nets) [1]. As the name suggests, WF-nets are used to model the ordering of tasks in workflow processes. The initial and final nodes indicate respectively the initial and final states of processed cases.

Definition 3. A Petri net $N$ is a Workflow net (WF-net) iff:

1. $N$ has two special places: $i$ and $f$. The initial place $i$ is a source place, $i . e .{ }^{\bullet} i=\emptyset$, and the final place $f$ is a sink place, i.e. $f^{\bullet}=\emptyset$.

2. For any node $n \in(P \cup T)$ there exists a path from $i$ to $n$ and a path from $n$ to $f$. (We call this property the path property of WF-nets.)

In this paper, we study the processing of batches of tasks in Workflow nets, meaning that the initial place of a Workflow net may contain an arbitrary number of tokens. Our goal is to provide correctness criteria for the design of these nets. One natural correctness requirement is proper termination, which is called soundness in the WF-net theory. We will use the generalised notion of soundness for WF-nets introduced in [4]:

Definition 4. We say that a WF-net $N$ with marking $m \in \mathcal{R}(k[i])$ terminates properly iff $m \stackrel{*}{\longrightarrow} k[f]$.

$N$ is $k$-sound for some $k \in \mathbb{N}$ iff for all $m \in \mathcal{R}(k[i]),(N, m)$ terminates properly. $N$ is sound iff it is $k$-sound for all $k \in \mathbb{N}$. 

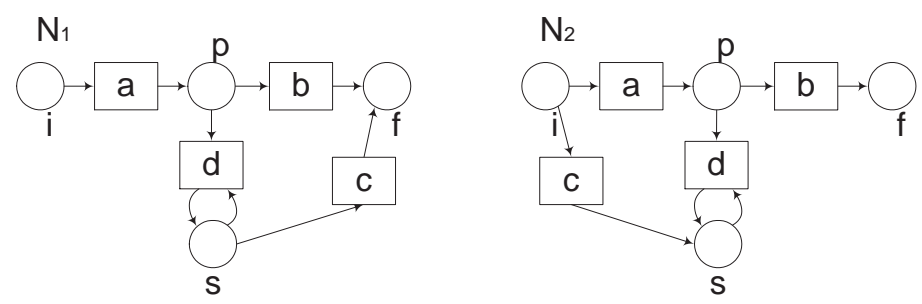

Fig. 1. Redundant and persistent places

We will use terms initial and final markings for markings $k[i]$ and $k[f]$ respectively $(k \in \mathbb{N})$. We will write $\mathbf{0}$ for the vector representation of marking $\emptyset$, $\mathbf{i}$ for the vector representation of marking $[i]$ and $\mathbf{f}$ for the vector representation of $[f]$. For every marking $m$ reachable from an initial marking $k[i]$ holds: $\mathcal{I} \cdot m=\mathcal{I} \cdot(k \cdot \mathbf{i})$, and if net $N$ with marking $m$ terminates properly, then $\mathcal{I} \cdot m=\mathcal{I} \cdot(k \cdot \mathbf{f})$.

\section{Batch Workflow Nets}

We are interested in the correct and optimal design of WF-nets. Ideally, correctness requirements should be formulated as requirements on the structure of the net (thus, they can be easily checked) and they should guarantee the correctness of the net behaviour. In this section, we consider behavioural criteria of the correct design and reduce them to structural ones.

\subsection{Structural Non-Redundancy for Workflow Nets}

Besides soundness, a logical requirement for the correct design of a WF-net is non-redundan$c y$, namely: every transition of the net can potentially fire and every place of the net can potentially obtain tokens, provided that there are enough tokens in the initial place. WFnet $N_{1}$ in Fig. 1 does not satisfy this requirement because transition $d$ can never fire and place $s$ can never get tokens. So $d$ and $s$ are redundant. At the same time, it should be possible for every place (except for $f$ ) to become unmarked again-otherwise the net is guaranteed to be not sound, as e.g. net $N_{2}$ in Fig. 1 - place $s$ can obtain tokens but it can never become unmarked after that, i.e. this place is persistent. In formal terms:

Definition 5. Let $N=\left\langle P, T, F^{+}, F^{-}\right\rangle$be a WF-net.

A place $p \in P$ is non-redundant iff there exist $k \in \mathbb{N}$ and $m \in \mathbb{N}^{P}$ such that $k[i] \stackrel{*}{\longrightarrow}$ $m \wedge p \in m$.

A place $p \in P$ is non-persistent iff there exist $k \in \mathbb{N}$ and $m \in \mathbb{N}^{P}$ such that $p \in m \wedge m \stackrel{*}{\longrightarrow}$ $k[f]$.

A transition $t$ is non-redundant iff there exist $k \in \mathbb{N}$ and $m \in \mathbb{N}^{P}$ such that $k[i] \stackrel{*}{\longrightarrow} m \stackrel{t}{\longrightarrow}$.

The following lemma presents these desirable behavioural properties in more general terms: 
Lemma 6. (1) A WF-net $N$ has no redundant places iff every marking is majorated by a marking reachable from some initial marking $k[i]$, i.e.

$\forall m \in \mathbb{N}^{P} \exists k \in \mathbb{N}, m^{\prime} \in \mathcal{R}(k[i]): m^{\prime} \geq m$.

(2) A WF-net $N$ has no persistent places iff every marking is majorated by a marking from which some final marking $k[f]$ is reachable, i.e.

$\forall m \in \mathbb{N}^{P}: \exists k \in \mathbb{N}, m^{\prime} \in \mathcal{S}(k[f]): m^{\prime} \geq m$.

Proof. (1) If every marking can be majorated by a marking reachable from some $k[i]$, then every marking $[p], p \in P$ can be majorated and $p$ is non-redundant. In the opposite direction: for every $p$ there exist $k_{p}, m_{p}$, such that $k_{p}[i] \stackrel{*}{\longrightarrow} m_{p}$ where $m_{p} \geq[p]$. Then we can majorate a given marking $m$ by a marking $m^{\prime}=\sum_{p \in m} m_{p}$ reachable from $\left(\sum_{p \in m} k_{p}\right)[i]$.

(2) can be proved similarly.

As an immediate consequence we obtain the following property:

Lemma 7. A WF-net $N$ has no redundant places iff it has no redundant transitions.

Proof. Let $N$ has no redundant places. Consider an arbitrary transition $t \in T$. By applying property (1) of Lemma 6 to $^{\bullet} t$ we obtain that $t$ can get enabled, and hence it is nonredundant.

Now assume that $N$ has no redundant transitions. Consider an arbitrary place $p \in P \backslash[i]$. Since $N$ is a WF-net, $\bullet p \neq \emptyset$, and since all transitions are non-redundant, transitions from - $p$ can fire and so $p$ can get marked. Thus $p$ is non-redundant.

Non-redundancy and non-persistency are behavioural properties. They imply though the following restrictions on the structure of the net: all proper siphons of the net should contain $i$ and all proper traps should contain $f$. If $N$ contained a proper siphon without $i$, the transitions consuming tokens from places of that siphon would be dead, no matter how many tokens are inserted into $i$. Similarly, if $N$ contained a trap without $f$, the net could not terminate properly. It is not surprising that the absence of traps and siphons is a necessary condition for the correctness of the design. What is more interesting is that the absence of such siphons and traps is a sufficient condition for the absence of redundant and persistent places respectively: if a net has a redundant place, there exists a proper siphon without $i$, and if a net has a persistent place, there exists a proper trap without $f$, i.e. these behavioural and structural characteristics are equivalent for WF-nets:

Theorem 8. Let $N=\left\langle P, T, F^{+}, F^{-}\right\rangle$be a WF-net. Then the following holds:

(1) $N$ has no redundant places iff $P \backslash\{i\}$ contains no proper siphon.

(2) $N$ has no persistent places iff $P \backslash\{f\}$ contains no proper trap.

Proof. (1) Let $X \subseteq P \backslash\{i\}$ be a proper siphon. Since an unmarked siphon stays unmarked, places from $X$ are redundant.

In the opposite direction: Let $X \subseteq P \backslash\{i\}$ be the set of all redundant places of $N$. We will prove that $X$ is a siphon. Consider some $t \notin X^{\bullet} ; \bullet^{\bullet} t$ contains no places from $X$ and hence all places from ${ }^{\bullet} t$ are non-redundant. Then for every place $p$ in $\bullet t$ there exists a 
input : A Petri net $N=\left(P, T, F^{+}, F^{-}\right)$and $S \subseteq P$;

output: $X \subseteq S$;

$X=S$;

while there exists $p \in X$ and $t \in \epsilon^{\bullet} p$ such that $t \notin X^{\bullet}$ do $X=X \backslash\{p\}$;

$\operatorname{return}(\mathrm{X})$;

Fig. 2. Algorithm for finding the maximal siphon in a set of places $S$

marking $m_{p} \geq[p]$ reachable from some $k_{p}[i], k_{p} \in \mathbb{N}$. Taking a sum of corresponding initial markings we obtain an initial marking from which a marking $m \geq{ }^{\bullet} t$ can be reached. Thus $t$ can fire and all places from $t^{\bullet}$ can obtain tokens, i.e. they are non-redundant. Therefore, $t^{\bullet} \cap X=\emptyset$ and so $t \notin \bullet X$. Hence $\left(T \backslash X^{\bullet}\right) \subseteq(T \backslash \bullet X)$, and so $X$ is a siphon. Thus every WF-net with redundant places contains a proper siphon in $P \backslash\{i\}$.

(2) can be proved analogously.

To check that $P \backslash\{i\}$ contains no proper siphon it is enough to compute the largest siphon $X$ in $P \backslash\{i\}$ in a standard manner [7] (see Fig.2): initialize $X$ with $P \backslash\{i\}$ and remove places that belong to $t^{\bullet}$ for some $t$ such that $t \notin X^{\bullet}$ until the fixed point is reached. The largest trap not containing $f$ can be computed with a similar algorithm.

As a spin-off of the check for absence of traps and siphons, we get a check of the path property of a WF-net:

Lemma 9. Let $N=\left\langle P, T, F^{+}, F^{-}\right\rangle$be a Petri net with a single source place $i$ and a single sink place $f$, and every transition of $N$ has at least one input and one output place. Moreover, $P \backslash\{i\}$ contains no proper siphon and $P \backslash\{f\}$ contains no trap. Then $N$ is a WF-net.

Proof. Consider an arbitrary node $n$ and the set $X$ of all places such that for every place $p \in X$ there is a path from $p$ to $n$. We will show that $X$ is a proper siphon, which implies that $i \in X$ and so there exists a path from $i$ to $n$.

The set $X$ is nonempty: If $n$ is a place then $n \in X$ (since there is a path from $n$ to $n$ ), and if $n$ is a transition then its input places are in $X$. $X$ it is a proper siphon: For every transition $t$, if $t \in \bullet^{\bullet} X$ then there is a path from $t$ to $n$, and hence there is a path from places from ${ }^{\bullet} t$ to $n$ and so every input place of $t$ is in $X$. Since $\bullet \neq \emptyset$, we have $t \in X^{\bullet}$. Thus, $X$ is a proper siphon indeed.

Similarly, the set of places to which there is a path from $n$ is a trap, and so there is a path from $n$ to $f$. Thus $N$ is indeed a WF-net.

Thus we obtained a characterization that guarantees non-redundancy and non-persistency for WF-nets, and moreover it serves as a check of the path property. 


\subsection{Batch Workflow nets}

Since we are interested in the class of WF-nets that have no redundant or persistent places, we introduce the notion of Batch Workflow nets by imposing requirements on the structure of the net:

Definition 10. A Batch Workflow net (BWF-net) $N$ is a Petri net that has the following properties:

(1) $N$ has a single source place $i$ and a single sink place $f$;

(2) every transition of $N$ has at least one input and one output place;

(3) every siphon of $N$ contains $i$;

(4) every trap of $N$ contains $f$.

The purpose of imposing structural requirements in the BWF-net definition resembles the purpose of one of the requirements on sound WF-nets from [1]. Sound WF-nets are defined there as nets where

(1) $\forall m \in \mathcal{R}([i]): m \stackrel{*}{\longrightarrow}[f]$,

(2) $\forall m \in \mathcal{R}([i]): m \geq[f] \Rightarrow m=[f]$

(3) $\forall t \in T: \exists m, m^{\prime}:[i] \stackrel{*}{\longrightarrow} m \stackrel{t}{\longrightarrow} m^{\prime}$.

Our definition of soundness is stronger than requirement (1) from the above definition: it corresponds to 1-soundness in our definition. We do not use requirement (2) since it follows immediately from (1) (we prove the implication in a generalized form that is applicable to nets with multiple tokens on $i$ ):

Lemma 11. Let $N$ be a WF-net such that $\forall m \in \mathcal{R}(k[i]): m \stackrel{*}{\longrightarrow} k[f]$. Then $\forall m \in$ $\mathcal{R}(k[i]): m \geq k[f] \Rightarrow m=k[f]$.

Proof. Consider a marking $m \in \mathcal{R}(k[i])$ such that $m \geq k[f]$, i.e. $m=m^{\prime}+k[f]$ for some $m^{\prime} \geq \emptyset$. Since $m \in \mathcal{R}(k[i]), m \stackrel{*}{\longrightarrow} k[f]$, i.e. $m^{\prime}+k[f] \stackrel{*}{\longrightarrow} k[f]$. Since $f^{\bullet}=\emptyset$, we have $m^{\prime} \stackrel{*}{\longrightarrow} \emptyset$. However, every transition of a WF-net has at least one output place. Thus $m^{\prime}=\emptyset$ and so $m=k[f]$.

We do not include requirement (3) in the definition of soundness. In fact we do not require all the transitions to be live in $(N,[i])$, since we allow batches of tasks to be processed in the net. The definition of BWF-nets implies that the net has no redundant transitions, which corresponds to (3).

In the rest of the paper we focus on the problem of soundness for BWF-nets. Working with BWF-nets instead of WF-nets does not limit the applicability of our approach: Let a WF-net $N$ be given. First, we find a maximal siphon $X$ in $P \backslash\{i\}$. All places from $X$ are redundant (see Theorem 8 ). Thus, transitions from $X^{\bullet}$ never get enabled and are redundant as well. Hence, by removing places from $X$ and transitions from $X^{\bullet}$ together with the corresponding ingoing and outgoing arcs we obtain a net $N_{1}$ with the same behavior as the one of net $N$, whatever initial marking $k[i]$ is chosen for these nets. $N_{1}$ is either not a WF-net any more (the path condition gets violated, or the place $f$ is removed) and so we can make the conclusion that $N$ was ill-designed, or $N_{1}$ is a WF-net with the same 
behavior as the original net $N$ but without redundant places. So $N_{1}$ is an improved version of $N$. Further, we check whether net $N_{1}$ has persistent places. If yes, we may conclude that $N_{1}$ is not a sound WF-net. Otherwise, $N_{1}$ is a BWF-net.

\section{Soundness is decidable}

In this section, we show that the problem of soundness is decidable for BWF-nets. As indicated before, this implies decidability of (general) soundness for WF-nets. We first discuss the necessary theoretical issues and then move to the actual decision procedure.

\subsection{Decidability of Soundness}

Proper termination for a given marking can be easily checked by using standard reachability algorithms. Deciding soundness is more intricate: A straightforward approach would require an infinite number of checks of proper termination (by checking proper termination for every marking reachable from some arbitrary initial marking). We shall try and reduce the check of soundness to the check of proper termination for a finite set of markings.

Consider the set of all markings reachable from some initial marking in a BWF-net $N$ : $\mathcal{R}=\bigcup_{k \in \mathbb{N}} \mathcal{R}(k \cdot \mathbf{i})$. Every $\mathcal{R}(k \cdot \mathbf{i})$ is a subset of the set

$$
\mathcal{G}_{k}=\left\{k \cdot \mathbf{i}+F \cdot v \mid v \in \mathbb{Z}^{T}\right\} \cap \mathbb{N}^{P}
$$

(see Lemma 1). Note that $\mathcal{R}(k \cdot \mathbf{i}) \subseteq \mathcal{G}_{k}$ but in general $\mathcal{G}_{k} \nsubseteq \mathcal{R}(k \cdot \mathbf{i})$. For every marking $m$ from $\mathcal{G}$ holds $\mathcal{I} \cdot m=\mathcal{I} \cdot(k \cdot \mathbf{i})(\mathcal{I}$ is a matrix with basis invariants as rows). Clearly, if a BWF-net is sound, then $\mathbf{i} \stackrel{*}{\longrightarrow} \mathbf{f}$, and hence $\mathcal{I} \cdot \mathbf{i}=\mathcal{I} \cdot \mathbf{f}$ (see Lemma 1 and Lemma 2). This is the first soundness check we perform. Further on, we will assume that $\mathcal{I} \cdot \mathbf{i}=\mathcal{I} \cdot \mathbf{f}$ holds for the net under consideration.

We now formulate the lemma that is fundamental for deciding soundness (in the proof we use the fact that $N$ is a $B W F-n e t$, and so it has no redundant places).

Lemma 12. Let $N$ be a sound BWF-net and let $m \in \mathcal{G}_{k}$ for some $k \in \mathbb{N}$. Then there exists $\ell \in \mathbb{N}$ such that $(k+\ell) \cdot \mathbf{i} \stackrel{*}{\longrightarrow} m+\ell \cdot \mathbf{f}$.

Proof. Let $m$ be a marking from $\mathcal{G}_{k}$, i.e. $m=k \cdot \mathbf{i}+F \cdot v$ for some $v \in \mathbb{Z}^{T}$. Then there exist $v_{1}, v_{2} \in \mathbb{N}^{T}$ such that $v=v_{1}-v_{2}$. Note that $F=F^{+}-F^{-}$. So

$$
m=k \cdot \mathbf{i}+F^{+} \cdot v_{1}+F^{-} \cdot v_{2}-F^{-} \cdot v_{1}-F^{+} \cdot v_{2} .
$$

By Lemma 6 , we can majorate markings $F^{+} \cdot v_{1}, F^{-} \cdot v_{2}$ : There exist $a, b \in \mathbb{N}$ and markings $A, B$ such that $a \cdot \mathbf{i} \stackrel{*}{\longrightarrow} A+F^{+} \cdot v_{1}$ and $b \cdot \mathbf{i} \stackrel{*}{\longrightarrow} B+F^{-} \cdot v_{2}$. Then $(k+a+b) \cdot \mathbf{i} \stackrel{*}{\longrightarrow}$ $k \cdot \mathbf{i}+A+F^{+} \cdot v_{1}+B+F^{-} \cdot v_{2}=m+A+F^{-} \cdot v_{1}+B+F^{+} \cdot v_{2}$.

Let $\gamma_{2}$ be an arbitrary firing sequence with $\overrightarrow{\gamma_{2}}=v_{2}$. Then $b \cdot \mathbf{i} \stackrel{*}{\longrightarrow} B+F^{-} \cdot v_{2} \stackrel{\gamma_{2}}{\longrightarrow} B+F^{+} \cdot v_{2}$, and since $N$ is sound, $B+F^{+} \cdot v_{2} \stackrel{*}{\longrightarrow} b \cdot \mathbf{f}$. Now consider a marking $A+F^{-} \cdot v_{1}$. For an 
arbitrary firing sequence $\gamma_{1}$ with $\overrightarrow{\gamma_{1}}=v_{1}, A+F^{-} \cdot v_{1} \stackrel{\gamma_{1}}{\longrightarrow} A+F^{+} \cdot v_{1}$. Moreover, we have $a \cdot \mathbf{i} \stackrel{*}{\longrightarrow} A+F^{+} \cdot v_{1}$, and since $N$ is sound, $A+F^{-} \cdot v_{1} \stackrel{*}{\longrightarrow} A+F^{+} \cdot v_{1} \stackrel{*}{\longrightarrow} a \cdot \mathbf{f}$. Thus we obtain $m+A+F^{-} \cdot v_{1}+B+F^{+} \cdot v_{2} \stackrel{*}{\longrightarrow} m+(a+b) \cdot \mathbf{f}$. So with $\ell=a+b$ the lemma holds.

This immediately leads us to the conclusion that every marking from $\mathcal{G}_{k}$ should enable some firing sequence leading to $k \cdot \mathbf{f}$, lest the soundness condition gets violated.

Lemma 13. Let $N$ be a sound $B W F$-net and $m \in \mathcal{G}_{k}$. Then $m \stackrel{*}{\longrightarrow} k \cdot \mathbf{f}$.

Proof. By Lemma 12, there exists $\ell \in \mathbb{N}$ such that $(k+\ell) \cdot \mathbf{i} \stackrel{*}{\longrightarrow} m+\ell \cdot \mathbf{f}$. Since $N$ is sound, $m+\ell \cdot \mathbf{f} \stackrel{*}{\longrightarrow}(k+\ell) \cdot \mathbf{f}$. Since $f$ is a sink place, we deduce $m \stackrel{*}{\longrightarrow} k \cdot \mathbf{f}$.

One more conclusion we can draw now is the following:

Corollary 14. Let $N$ be a sound BWF-net. Then $\mathcal{I} \cdot x=\mathbf{0}$ for $x \in\left(\mathbb{Q}^{+}\right)^{P}$ iff $x=\mathbf{0}{ }^{1}$

Proof. Let $\mathcal{I} \cdot x=\mathbf{0}$ for some $x>\mathbf{0}$. Then we can find $\ell \in \mathbb{N}$ such that $y=\ell \cdot x$ is in $\mathbb{N}^{P}$. Note that $\mathcal{I} \cdot y=0$ as well. So $y$ agrees with marking $\mathbf{0}$ on all place invariants. By Lemma $2, y=F \cdot v$ for some $v \in \mathbb{Q}^{T}$. Then there exists $n>0$ such that $w=n \cdot v \in \mathbb{Z}^{T}$ and thus $z=n \cdot y=F \cdot w$ is also the solution for the equation $\mathcal{I} \cdot x=\mathbf{0}$. Note that $z$ is a non-empty marking from $\mathcal{G}_{0}$. By Lemma 13 , we have $z \stackrel{*}{\longrightarrow} \mathbf{0}$. This is in contradiction with the fact that for any transition of $N, t^{\bullet} \neq \emptyset$.

Thus, the second soundness check we perform is the check whether the equation $\mathcal{I} \cdot x=\mathbf{0}$ has only the trivial solution on $\mathbb{N}^{P}$. If so, we can conclude that $\mathcal{G}_{k}$ 's are disjoint sets:

Corollary 15. Let $N$ be a $B W F$-net such that the equation $\mathcal{I} \cdot x=\mathbf{0}$ has only the trivial solution in $\mathbb{N}^{P}$. Then $\mathcal{G}_{k} \cap \mathcal{G}_{\ell} \neq\{\mathbf{0}\}$ implies $k=\ell$.

Proof. Since $m \in \mathcal{G}_{k}$ implies $\mathcal{I} \cdot m=\mathcal{I} \cdot(k \cdot \mathbf{i})$ and $m \in \mathcal{G}_{\ell}$ implies $\mathcal{I} \cdot m=\mathcal{I} \cdot(\ell \cdot \mathbf{i})$, $m \in\left(\mathcal{G}_{k} \cap \mathcal{G}_{\ell}\right)$ implies $\mathcal{I} \cdot(k \cdot \mathbf{i})=\mathcal{I} \cdot(\ell \cdot \mathbf{i})$. By Corollary $14, k=\ell$.

Further on, we assume that $\mathcal{I} \cdot x=\mathbf{0}$ has only the trivial solution on $\mathbb{N}^{P}$ and thus $\mathcal{G}_{k}$ 's are disjoint sets. We define the $i$-weight function $w(m)$ of a marking $m$ as a natural number $k$ such that $\mathcal{I} \cdot m=\mathcal{I} \cdot(k \cdot \mathbf{i})(w(m)$ is undefined if such a value $k$ does not exist). All markings in $\mathcal{G}_{k}$ have $i$-weight $k$.

Now we introduce the set $\mathcal{G}=\bigcup_{k \in \mathbb{N}} \mathcal{G}_{k}$, i.e.

$$
\mathcal{G}=\left\{k \cdot \mathbf{i}+F \cdot v \mid k \in \mathbb{N} \wedge v \in \mathbb{Z}^{T}\right\} \cap \mathbb{N}^{P},
$$

and extend the notion of proper termination for all markings from $\mathcal{G}$ : We say that a marking $m \in \mathcal{G}$ terminates properly iff $m \stackrel{*}{\longrightarrow} w(m) \cdot \mathbf{f}$.

Lemma 16. Let $m_{1}, m_{2} \in \mathcal{G}$ be markings that terminate properly and $m=\lambda_{1} m_{1}+\lambda_{2} m_{2}$ for some $\lambda_{1}, \lambda_{2} \in \mathbb{N}$. Then $m \in \mathcal{G}$ and it terminates properly.

\footnotetext{
${ }^{1}$ We overload the notation and use $\mathbf{0}$ for a zero-vector of an arbitrary dimension.
} 
Proof. By the definition of $\mathcal{G}_{k},\left(\lambda_{1} m_{1}+\lambda_{2} m_{2}\right) \in \mathcal{G}_{\lambda_{1} k_{1}+\lambda_{2} k_{2}}$ and $\mathcal{I} \cdot\left(\lambda_{1} m_{1}+\lambda_{2} m_{2}\right)=$ $\mathcal{I} \cdot\left(\left(\lambda_{1} k_{1}+\lambda_{2} k_{2}\right) \cdot \mathbf{i}\right)$. Thus $w\left(\lambda_{1} m_{1}+\lambda_{2} m_{2}\right)=\lambda_{1} w\left(m_{1}\right)+\lambda_{2} w\left(m_{2}\right)$. Since $m_{1} \stackrel{*}{\longrightarrow} w\left(m_{1}\right) \cdot \mathbf{f}$ and $m_{2} \stackrel{*}{\longrightarrow} w\left(m_{2}\right) \cdot \mathbf{f}$, we have $\lambda_{1} m_{1}+\lambda_{2} m_{2} \stackrel{*}{\longrightarrow}\left(\lambda_{1} w\left(m_{1}\right)+\lambda_{2} w\left(m_{2}\right)\right) \cdot \mathbf{f}$.

We now formulate a necessary and sufficient condition for soundness.

Theorem 17. Let $N$ be a BWF-net. Then $N$ is sound iff all markings in $\mathcal{G}$ terminate properly.

Proof. $(\Rightarrow)$ : Suppose $N$ is sound. Consider arbitrary $k \in \mathbb{N}, m \in \mathcal{G}_{k}$. By Lemma 13, $m \stackrel{*}{\longrightarrow} k \cdot \mathbf{f}$.

$(\Leftarrow)$ : Suppose $\forall k \in \mathbb{N}, m \in \mathcal{G}_{k}: m \stackrel{*}{\longrightarrow} k \cdot \mathbf{f}$. Let $k \cdot \mathbf{i} \stackrel{*}{\longrightarrow} m$ for some $k, m$. Then $m \in \mathcal{G}_{k}$ and therefore $m \stackrel{*}{\longrightarrow} k \cdot \mathbf{f}$. Hence $N$ is sound.

We thus obtained a characterization of soundness involving the set $\mathcal{G}$ rather than reachable markings. We shall use the regularity of the structure of $\mathcal{G}$ to reduce the problem of proper termination of markings of $\mathcal{G}$ to the problem of proper termination of some finite subset $\Gamma$ of $\mathcal{G}$.

In order to construct $\Gamma$, we extend the set $\mathcal{G}$ even further by making a step from integers to rational numbers and considering the set

$$
\mathcal{H}=\left\{a \cdot \mathbf{i}+F \cdot v \mid a \in \mathbb{Q}^{+} \wedge v \in \mathbb{Q}^{T}\right\} \cap\left(\mathbb{Q}^{+}\right)^{P} .
$$

(We refer to the appendix for the definitions of algebraic notions that we use in the rest of this section.)

Lemma 18. The set $\mathcal{H}$ is a convex polyhedral cone. Moreover, there exists a finite set $e_{1}, \ldots, e_{n}$ of generators such that $e_{1}, \ldots, e_{n} \in \mathcal{G}$.

Proof. $\mathcal{H}$ is a convex polyhedral cone. By Theorem 26, we can find generators $E_{1}, \ldots, E_{n}$ of $\mathcal{H}$. Each $E_{i}$ is a linear combination of $\mathbf{i}$ and the column vectors of $F$ with rational coefficients. The lcm of the denominators divided by the ged of the numerators gives for a given $E_{i}$ the smallest rational number $\gamma_{i}$ such that $\gamma_{i} E_{i}$ can be written as $k_{i} \cdot \mathbf{i}+F \cdot v_{i}$ with $k_{i} \in \mathbb{N}, v_{i} \in \mathbb{Z}^{T}$. Set $e_{i}=\gamma_{i} E_{i}$ for $i=1, \ldots, n$. Then the $e_{1}, \ldots, e_{n}$ are generators of $\mathcal{H}$ and $e_{1}, \ldots, e_{n} \in \mathcal{G}$.

We define our finite set $\Gamma$ as

$$
\Gamma=\left\{\sum_{i} \alpha_{i} \cdot e_{i} \mid 0 \leq \alpha_{i} \leq 1\right\} \cap \mathcal{G}
$$

and show that the proper termination of any marking from $\mathcal{G}$ can be reduced to the proper termination of markings from $\Gamma$. Note that $e_{1}, \ldots, e_{n}$ are in $\Gamma$.

Theorem 19. Let $N$ be a $B W F-n e t$ such that $\mathcal{I} \cdot \mathbf{i}=\mathcal{I} \cdot \mathbf{f}$ and $\mathcal{I} \cdot x=\mathbf{0}$ has only the trivial solution in $\left(\mathbb{Q}^{+}\right)^{P}$. Further, let $\mathcal{G}=\left\{k \cdot \mathbf{i}+F \cdot v \mid k \in \mathbb{N} \wedge v \in \mathbb{Z}^{T}\right\} \cap \mathbb{N}^{P}$, $\mathcal{H}=\left\{a \cdot \mathbf{i}+F \cdot v \mid a \in \mathbb{Q}^{+} \wedge v \in \mathbb{Q}^{T}\right\} \cap\left(\mathbb{Q}^{+}\right)^{P}, e_{1}, \ldots, e_{n} \in \mathcal{G}$ be the generators of the cone $\mathcal{H}$ and $\Gamma=\left\{\sum_{i} \alpha_{i} \cdot e_{i} \mid 0 \leq \alpha_{i} \leq 1\right\} \cap \mathcal{G}$. Then $N$ is sound iff all markings from $\Gamma$ terminate properly. 
Proof. $(\Rightarrow)$ : Let $N$ be a sound WF-net. By Theorem 17 all markings of $\mathcal{G}$ terminate properly. Since $\Gamma \subseteq \mathcal{G}$, all markings of $\Gamma$ terminate properly.

$(\Leftarrow)$ : Let all markings from $\Gamma$ terminate properly. Consider an arbitrary marking $m \in \mathcal{G}$. Since $m \in \mathcal{G}, m=n \cdot \mathbf{i}+F \cdot w$ for some $n \in \mathbb{N}, w \in \mathbb{Z}^{T}$. Since $\mathcal{G} \subseteq \mathcal{H}$ and so $m \in \mathcal{H}$, $m=\sum_{i} \lambda_{i} \cdot e_{i}$ with the $\lambda_{i} \in \mathbb{Q}^{+}$. We can represent $m$ as $\sum_{i} \ell_{i} \cdot e_{i}+\sum_{i} \mu_{i} \cdot e_{i}$ where $\ell_{i}=\left\lfloor\lambda_{i}\right\rfloor$ (the integer part of $\lambda_{i}$ ) and $\mu_{j}=\lambda_{j}-\left\lfloor\lambda_{j}\right\rfloor$ (the fractional part of $\lambda_{i}$ ), i.e. $0 \leq \mu_{i}<1$. We will prove that $m^{\prime}=m-\sum_{i} \ell_{i} \cdot e_{i}=\sum_{i} \mu_{i} \cdot e_{i}$ is a marking from $\mathcal{G}$. First note that $m \in \mathbb{N}^{P}, e_{i} \in \mathbb{N}^{P}, \ell_{i} \in \mathbb{N}$ for all $i$. Thus $m^{\prime} \in \mathbb{Z}^{P}$. Moreover, $\mu_{i} \geq 0$, which implies that $m^{\prime} \geq \mathbf{0}$. Thus $m^{\prime} \in \mathbb{N}^{P}$. Since $e_{i} \in \mathcal{G}$, they can be represented as $k_{i} \cdot \mathbf{i}+F \cdot v_{i}$ where $k_{i} \in \mathbb{N}, v_{i} \in \mathbb{Z}^{T}$. Since $m^{\prime}=m-\sum_{i} \ell_{i} \cdot e_{i}$, we have $m^{\prime}=k \cdot \mathbf{i}+F \cdot v$ with $k=n-\sum_{i} k_{i} \cdot \ell_{i}$ and $v=w-\sum_{i} \ell_{i} \cdot v_{i}$. Therefore, we can conclude that $k \in \mathbb{Z}$ and $v \in \mathbb{Z}^{T}$. Now we only have to show that $k \in \mathbb{N}$.

Note that since columns of $F$ are not necessarily linearly independent, the representation of $m^{\prime}$ as $k \cdot \mathbf{i}+F \cdot v$ is not necessarily unique. However, $k$ has the same value in every such a representation: Let $a=\mathcal{I} \cdot m^{\prime}$ and $k \cdot \mathbf{i}+F \cdot v$ is some representation of $m^{\prime}$. Then $\mathcal{I} \cdot m^{\prime}=\mathcal{I} \cdot(k \cdot \mathbf{i})+\mathcal{I} \cdot(F \cdot v)$. By the definition of place invariants, $\mathcal{I} \cdot(F \cdot v)=0$. Thus $a=\mathcal{I} \cdot(k \cdot i)$. Due to Corollary $14, k$ is uniquely defined. Since $k=\sum_{i} \mu_{i} \cdot k_{i}, k \geq 0$ and so $k \in \mathbb{N}$. Therefore $m^{\prime} \in \mathcal{G}$. Since $0 \leq \mu_{i}<1, m^{\prime} \in \Gamma$.

Since $e_{1}, \ldots, e_{n}$ and $m^{\prime}$ are markings from $\Gamma$ and thus terminate properly, $m$ terminates properly as well (Lemma 16). Thus, all markings in $\mathcal{G}$ terminate properly and, due to Theorem 17, $N$ is sound.

Thus we have reduced the problem of soundness to the problem of proper termination of a finite number of markings and hereby proved its decidability.

\subsection{Decision Procedure}

In this subsection, we describe the decision procedure for soundness in a systematic way. We do not claim algorithm status: we focused on clarity rather than efficiency here.

We start with trivial checks that can lead to the negative answer. First we find a set of basis place invariants and check that $\mathcal{I} \cdot \mathbf{i}=\mathcal{I} \cdot \mathbf{f}$. If not, the net is not sound. Then we check that the only solution of the equation $\mathcal{I} \cdot x=\mathbf{0}$ on $\left(\mathbb{Q}^{+}\right)^{P}$ is the trivial solution $x=\mathbf{0}$; otherwise the net is not sound (see Corollary 14). This second condition also guarantees the boundedness of the net:

Lemma 20. Let $N$ be a BWF-net such that the only solution of $\mathcal{I} \cdot x$ in $\left(\mathbb{Q}^{+}\right)^{P}$ is $x=0$. Then $\mathcal{R}(k \cdot \mathbf{i})$ and $\mathcal{S}(k \cdot \mathbf{f})$ are finite sets for any $k \in \mathbb{N}$.

Proof. Assume some $\mathcal{R}(k \cdot \mathbf{i})$ is an infinite set. Then there exist $m_{1}, m_{2} \in \mathcal{R}(k \cdot \mathbf{i})$ such that $m_{1}<m_{2}$ and $\mathcal{I} \cdot m_{1}=\mathcal{I} \cdot m_{2}=\mathcal{I} \cdot(k \cdot \mathbf{i})$. Then $\mathcal{I} \cdot\left(m_{2}-m_{1}\right)=\mathbf{0}$ and thus $m_{2}-m_{1}=\mathbf{0}$. This is a contradiction with $m_{1}<m_{2}$. Thus $\mathcal{R}(k \cdot \mathbf{i})$ is finite. Analogously, we prove that $\mathcal{S}(k \cdot \mathbf{f})$ is a finite set.

At the next step, we compute generators $E_{1}, \ldots, E_{n}$ of $\mathcal{H}$ and rescale them to obtain generators $e_{1}, \ldots, e_{n} \in \mathcal{G}$ of $\mathcal{H}$. Now our goal is to enumerate the markings of $\Gamma$. 
The generators of the cone $\mathcal{H}$ are not necessarily linearly independent (e.g. the set of generators can include vectors $e, f, g$ and $f+g-e$ ). This implies that the representation of elements of the cone as nonnegative linear combinations of the cone generators are not necessarily unique. However, by Carathéodory's theorem (Theorem 27 in Appendix A), we can represent $\mathcal{H}$ as $\mathcal{H}=\bigcup_{j} \mathcal{H}_{j}$, where $\mathcal{H}_{j}$ 's are cones generated by vectors from some maximal subset $\mathcal{E}_{j} \subseteq\left\{e_{1}, \ldots, e_{n}\right\}$ of linearly independent vectors. We define $\mathcal{G}_{j} \subseteq \mathcal{G}$ as $\mathcal{G}_{j}=\left(\mathcal{G} \cap \mathcal{H}_{j}\right)$ and $\Gamma_{j} \subseteq \Gamma$ as

$$
\Gamma_{j}=\left\{\sum_{e_{i} \in \mathcal{E}_{j}} \lambda_{i} e_{i} \mid 0<\lambda_{i} \leq 1\right\} \cap \mathcal{G}_{j}
$$

Note that $\mathcal{G}=\bigcup_{j} \mathcal{G}_{j}$ but in general $\Gamma \neq \bigcup_{j} \Gamma_{j}$ though $\Gamma \supseteq \bigcup_{j} \Gamma_{j}$. Still, the proper termination of markings from $\bigcup_{j} \Gamma_{j}$ guarantees the proper termination of markings in $\mathcal{G}$. We do not give a complete proof but sketch the main idea. Every $x \in \mathcal{G}$ is also an element of $\mathcal{H}$ and so an element of some $\mathcal{H}_{j}$. Thus it can be represented as $\sum_{e_{i} \in \mathcal{E}_{j}} \lambda_{i} \cdot e_{i}$. Now we can use the same construction with the integer and the fractional part of $\lambda_{i}$ as in the proof of Theorem 19 to prove that $x$ is a nonnegative integer linear combination of markings that terminate properly, $x$ terminates properly as well.

Now we will construct an algorithm for the enumeration of the elements of $\Gamma_{j}$. Since vectors in $\mathcal{E}_{j}$ are linearly independent, any $x \in \mathcal{G}_{j}$ has a unique representation as a nonnegative linear combination of vectors from $\mathcal{E}_{j}$. Note that the dimension $m$ of any set $\mathcal{E}_{i}$ equals the dimension of the vector space $\left\{\lambda \cdot \mathbf{i}+F \cdot v \mid \lambda \in \mathbb{Q} \wedge v \in \mathbb{Q}^{T}\right\}$, thus all vectors from this vector space have unique representations as $\sum_{e_{i} \in \mathcal{E}_{i}} \lambda_{i} \cdot e_{i}$ where $\lambda_{i} \in \mathbb{Q}$. Now consider the set $\mathcal{U} \supseteq \mathcal{G}_{j}$ defined as $\mathcal{U}=\left\{k \cdot \mathbf{i}+F \cdot v \mid k \in \mathbb{N} \wedge v \in \mathbb{Z}^{T}\right\}$ (note that we do not require elements of $\mathcal{U}$ to be nonnegative vectors). Every element of $\mathcal{U}$ has a unique representation as a linear combination of vectors from $\mathcal{E}_{j}$. We define the translation functions $\theta_{j}: \mathcal{U} \rightarrow \Gamma_{j}$ as follows:

$$
\theta_{j}\left(\sum_{e_{i} \in \mathcal{E}_{j}} \lambda_{i} \cdot e_{i}\right)=\sum_{e_{i} \in \mathcal{E}_{j}} \mu_{i} \cdot e_{i} \text { where } \mu_{i}=\lambda_{i}-\left\lfloor\lambda_{i}\right\rfloor\left(\text { the fractional part of } \lambda_{i}\right) .
$$

We will use a simple property of the translation function:

Lemma 21. For any markings $x, y \in \mathcal{H}_{i}, \theta_{j}(x+y)=\theta_{j}\left(x+\theta_{j}(y)\right)$.

Lemma 22. $\Gamma_{j}=\left\{\theta_{j}(x) \mid x \in \mathcal{U}\right\}$.

Proof. $\Gamma_{j} \subseteq \mathcal{G}_{j} \subseteq \mathcal{U}$ and for any $x \in \Gamma_{j}, \theta_{j}(x)=x$ (by the definition of $\theta$ ), so $\Gamma_{j} \subseteq\left\{\theta_{j}(x) \mid\right.$ $x \in \mathcal{U}\}$.

For every $x \in \mathcal{G}_{j}, \theta_{j}(x) \in \Gamma_{j}$ (cf. the proof of Theorem 19).

For any $x \in\left(\mathcal{U} \backslash \mathcal{G}_{j}\right)$, there exist representations of $x$ as $k \cdot \mathbf{i}+F \cdot v$ where $k \in \mathbb{N}, v \in \mathbb{Z}^{T}$ and as $\sum_{i} \lambda_{i} e_{i}$ with $e_{i} \in \mathcal{E}_{i}$ and $\lambda_{i} \in \mathbb{Q}$. We choose $y \in \mathcal{G}_{j}, y=\ell \cdot \mathbf{i}+F \cdot w=\sum_{i} \alpha_{i} e_{i}$ with $\ell \in \mathbb{N}, w \in \mathbb{Z}^{T}, \alpha \in \mathbb{N}, e_{i} \in \mathcal{E}_{i}$, such that $k+\ell \geq 0$ and $\alpha_{i}+\lambda_{i} \geq 0$ for all $i$. Now consider the marking $z=x+y$. By the choice of $y, z \geq \mathbf{0}$ and $z=(k+\ell) \cdot \mathbf{i}+F \cdot(v+w)$ 
input : a BWF-net $N$, a set of linearly independent vectors $\mathcal{E}_{i}$, translation function $\theta_{j}$;

output : the set $X$ of markings;

$X=\{\mathbf{0}\}$

repeat

$X=X \cup\left\{\theta_{j}(x+\mathbf{i}) \mid x \in X\right\} \cup\left\{\theta_{j}\left(x \pm F_{t}\right) \mid x \in X \wedge t \in T\right\}$

until the fixed point is reached;

$\operatorname{return}(X)$;

Fig. 3. Algorithm for enumeration of $\Gamma$

with $(k+l) \in \mathbb{N}$ and $(v+w) \in \mathbb{Z}^{T}$, i.e. $z \in \mathcal{G}$. Moreover, $z=\sum_{i}\left(\alpha_{i}+\lambda_{i}\right) \cdot e_{i}$, i.e. it is a nonnegative linear combination of vectors from $\mathcal{E}_{i}$ and thus $z \in \mathcal{G}_{j}$. Note that $\theta_{j}(y)=0$, which means $\theta_{j}(z)=\theta_{j}(x)$. Since $z \in \mathcal{G}_{j}, \theta_{j}(z) \in \Gamma_{j}$ and so $\theta_{j}(x) \in \Gamma_{j}$. Thus $\Gamma_{j} \supseteq\left\{\theta_{j}(x) \mid x \in \mathcal{U}\right\}$.

Fig. 3 gives the algorithm that enumerates the elements of $\Gamma_{j} . F_{t}$ stands there for the column of $F$ that corresponds to transition $t$, i.e. that is a vector corresponding to $t^{\bullet}-{ }^{\bullet} t$.

Theorem 23. The algorithm in Fig. 3 terminates and its output equals $\Gamma_{j}$.

Proof. First we prove by induction on the iteration step number that $X \subseteq \Gamma_{j}$ at every iteration step. Let $X_{\ell}$ be the value of $X$ at iteration $l . X_{0}=\{\mathbf{0}\}$ and by the definition of $\Gamma_{j}, \mathbf{0} \in \Gamma_{j}$. Now let $X_{\ell} \subseteq \Gamma_{j}$ at some step. Consider some $x \in X_{\ell}$ (and hence $x \in \Gamma_{j}$ and $x \in \mathcal{U})$. The elements added to $X$ at iteration $(\ell+1)$ are $(x+\mathbf{i})$ and $\left(x \pm F_{t}\right), t \in T$. By the definition of $\mathcal{U},(x+\mathbf{i}) \in \mathcal{U}$ and thus $\theta_{j}(x+\mathbf{i}) \in \Gamma_{j}$ by the definition of $\theta_{j}$. Now consider a marking $\left(x \pm F_{t}\right), t \in T$. By the definition of $\mathcal{U},\left(x \pm F_{t}\right) \in \mathcal{U}$ and so $\theta_{j}\left(x \pm F_{t}\right) \in \Gamma_{j}$. Thus, $X \subseteq \Gamma_{j}$ at every iteration step. Since $X$ grows monotonously and $\Gamma_{j}$ is bounded, the algorithm terminates and it returns a subset $X$ of $\Gamma_{j}$.

Now we will prove that $\Gamma_{j} \subseteq X$. Any marking $x \in \Gamma_{j}$ is a marking from $\mathcal{U}$ and hence it can be represented as $m=k \cdot \mathbf{i}+F \cdot v$ for some $k \in \mathbb{N}$ and $v \in \mathbb{Z}^{T}$ with components $v_{1}, \ldots, v_{n} \in \mathbb{Z}$. We define $\|x\|=k+\sum_{j}\left|v_{j}\right|$. Then for any $x$ such that $\|x\| \leq l, \theta_{j}(x) \in X_{l}$. We prove it by induction on $l$. For $l=0$ it holds trivially. Let it hold for some $l$. Consider $x=k \cdot \mathbf{i}+F \cdot v$ such that $\|x\|=l+1$. If $k>0, x=\mathbf{i}+((k-1) \cdot \mathbf{i}+F \cdot v)$. Note that for $y=(k-1) \cdot \mathbf{i}+F \cdot v,\|y\|=l$ and thus $\theta_{j}(y) \in X_{l}$. By Lemma $21, \theta_{j}(x)=\theta_{j}(\mathbf{i}+y)=$ $\theta_{j}\left(\mathbf{i}+\theta_{j}(y)\right)$. Since $\theta_{j}(y) \in X_{l}, \theta_{j}\left(\mathbf{i}+\theta_{j}(y)\right)$ is in $X_{l+1}$ by the definition of the algorithm, and thus $\theta_{j}(x) \in X_{l+1}$.

If $k=0$ then $v \neq \mathbf{0}$, i.e. $v_{j} \neq 0$ for some $j$. We represent $x$ as $F_{t_{j}}+\left(k \cdot \mathbf{i}+F \cdot v^{\prime}\right)$ where all components of $v^{\prime}$ equal the corresponding components of $v$, except for $v_{j}^{\prime}$ : $\left|v_{j}^{\prime}\right|=\left|v_{j}\right|-1$ and $v_{j} \cdot v_{j}^{\prime} \geq 0$. Then we use a similar induction scheme to prove the statement.

Thus at iteration $l=\max _{x \in \Gamma_{j}}\|x\|, X_{l}$ contains all elements of $\Gamma_{j}$.

When we found all markings of $\Gamma_{j}$ 's, we check proper termination for every marking. To check whether a marking $m$ terminates properly, it is enough to check whether $m \in$ $\mathcal{S}(w(m) \cdot \mathbf{f})$. Since $\mathcal{S}(w(m) \cdot \mathbf{f})$ is a finite set (see Lemma 20), we can construct it by a 


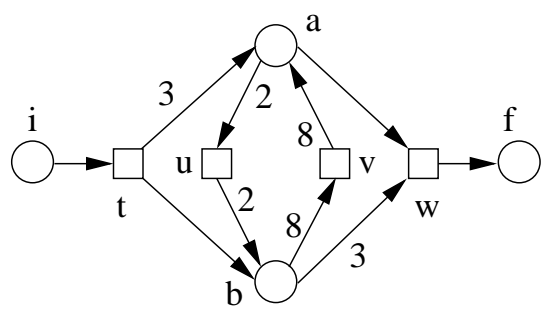

Fig. 4. Example net $N$

backward reachability analysis, starting with $X=\{w(m) \cdot \mathbf{f}\}$ and consequently augmenting this set by adding markings $\left\{x-F_{t} \mid x \in X \wedge t \in T \wedge\left(x-F_{t}\right) \geq \mathbf{0}\right\}$.

\section{Example}

We illustrate the decision procedure for soundness with an example. Consider net $N$ depicted in Figure 4. (The net has weighted arcs as indicated.) We have $P=\{i, a, b, f\}, T=$ $\{t, u, v, w\}$ and the incidence matrix

$$
F=\left(\begin{array}{rrrr}
-1 & 0 & 0 & 0 \\
3 & -2 & 8 & -1 \\
1 & 2 & -8 & -3 \\
0 & 0 & 0 & 1
\end{array}\right)
$$

First, we find the place invariants of the net. Solutions of the equation $I \cdot F=0$ are vectors $I=k \cdot(4,1,1,4), k \in \mathbb{Q}$. Thus the matrix of basis invariants $\mathcal{I}$ is $(4,1,1,4)$. Now we check that $\mathcal{I} \cdot \mathbf{i}=\mathcal{I} \cdot \mathbf{f}$ indeed (the first and the last columns of $\mathcal{I}$ coincide).

The cone $\mathcal{H}$ for this example is the set $\left(\mathbb{Q}^{+}\right)^{P}$, i.e. $\mathcal{H}$ is the cone generated by vectors $\mathbf{i}, \mathbf{a}, \mathbf{b}, \mathbf{f}$ corresponding to markings $[i],[a],[b],[f]$. These are vectors from $\mathcal{H}$, so they are representable as $a \cdot \mathbf{i}+F \cdot v$ for some $a \in \mathbb{Q}^{+}, v \in \mathbb{Q}^{T}$. By solving linear equations, we obtain the following representations of $\mathbf{a}$ and $\mathbf{b}$.

$$
\mathbf{a}=\frac{1}{4} \cdot \mathbf{i}+F \cdot\left(\begin{array}{c}
1 / 4 \\
1 / 8 \\
0 \\
0
\end{array}\right) \text { and } \mathbf{b}=\frac{1}{4} \cdot \mathbf{i}+F \cdot\left(\begin{array}{c}
1 / 4 \\
5 / 8 \\
0 \\
0
\end{array}\right)
$$

To find generators from $\mathcal{G}$ we need to rescale these vectors. Thus, we obtain $e_{1}=\mathbf{i}, e_{2}=$ $8 \cdot \mathbf{a}, e_{3}=8 \cdot \mathbf{b}, e_{4}=\mathbf{f}$. So we have defined our set $\Gamma$ and we can find all the points inside it. In Figure 5, the points of $\Gamma$ are depicted, projected on the $(a, b)$-plane. We have also depicted the transitions between them, from which it can be inferred that all grid points terminate. The net $N$ is thus sound.

If the transition $v$ were removed from $N$, the resulting net would not be sound, since the grid point $e_{3}$ no longer terminates. The net then is 1-sound but not 2-sound. 


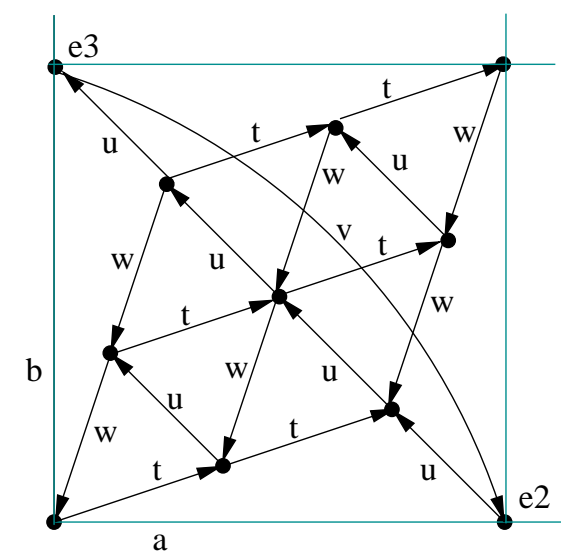

Fig. 5. Grid within $\Gamma$ of net $N$

\section{Conclusion}

We have introduced a subclass of Workflow nets: Batch Workflow nets (BWF-nets) that have a simple structural characterisation based on traps and siphons. Batch Workflow nets are Workflow nets without redundant places and transitions and without persistent places. We have shown that Workflow nets with redundant places/transitions or with persistent places are ill-designed. Therefore, we moved from the study of Workflow nets to the study of Batch Workflow nets.

Since we are interested in the processing of batches of tasks in the net, we investigated the generalised notion of soundness introduced in [4] for Batch Workflow nets. We have proved that the generalised soundness is decidable and have described the decision procedure.

The decidability of soundness implies trivially the decidability of weak separability [4]. It remains still unclear whether strong separability is decidable.

Future work For the soundness decision procedure, we focused on the clarity rather than efficiency. It is still to be investigated how to solve the problem of soundness in an efficient manner and what complexity this algorithm would have. Besides, soundness preserving Petri net reduction techniques can be employed prior to the use of the soundness decision procedure to speed up the check.

Acknowledgements We want to thank our colleague Aart Blokhuis for useful discussions on algebraic issues.

\section{References}

1. W. van der Aalst. Verification of workflow nets. In P. Azéma and G. Balbo, editors, Application and Theory of Petri Nets 1997, ICATPN'1997, volume 1248 of Lecture Notes in Computer Science. Springer-Verlag, 1997. 
2. F. Commoner. Deadlocks in Petri Nets. Applied Data Research, Inc., Wakefield, Massachusetts, Report CA7206-2311, 1972.

3. J. Desel and J. Esparza. Free Choice Petri nets., volume 40 of Cambridge Tracts in Theoretical Computer Science. Cambridge University Press, 1995.

4. K. van Hee, N. Sidorova, and M. Voorhoeve. Soundness and separability of workflow nets in the stepwise refinement approach. In W. van der Aalst and E. Best, editors, Application and Theory of Petri Nets 2003, ICATPN'2003, volume 2679 of Lecture Notes in Computer Science. Springer-Verlag, 2003.

5. K. Lautenbach. Liveness in Petri Nets. Internal Report of the Gesellschaft für Mathematik und Datenverarbeitung, Bonn, Germany, ISF/75-02-1, 1975.

6. A. Schrijver. Theory of Linear and Integer Programming. Wiley-Interscience series in discrete mathematics. John Wiley \& Sons, 1986.

7. P. Starke. Analyse von Petri-Netz-Modellen. Teubner, 1990.

8. H. Verbeek, T. Basten, and W. van der Aalst. Diagnosing workflow processes using woflan. The Computer Journal, 44(4):246-279, 2001.

\section{A Appendix}

Here we give the mathematical definitions and results we need (see e.g. [6] for more detail). Let $E_{n}$ stand for an $n$-dimensional vector space over some number field (in our case the rational numbers). Let $u$ be a vector. We write $u(x)$ for the value of the vector component corresponding to coordinate $x$.

A convex combination of the vectors $u_{1}, u_{2}, \ldots, u_{n}$ from $E_{n}$ is a vector $u=\alpha_{1} \cdot u_{1}+\alpha_{2}$. $u_{2}+\ldots+\alpha_{n} \cdot u_{n}$ where the $\alpha_{i}$ are nonnegative scalars such that $\sum_{i} \alpha_{i}=1$. A subset $S$ of $E_{n}$ is convex iff for all pairs of vectors $u_{1}, u_{2}$ any convex combination of them is also in $S$.

Lemma 24. The intersection of two convex sets is convex.

A set of vectors $C$ is called a cone if, for every vector $u \in C, \lambda u \in C$ for every nonnegative $\lambda$. A convex cone $C$ is polyhedral if $C=\{x \mid A \cdot x \leq 0\}$ for some matrix $A$, i.e. $C$ is the intersection of finitely many linear half-spaces.

Lemma 25. Let $X, Y$ be convex polyhedral cones. Then $Z=X \cap Y$ is a convex polyhedral cone as well.

Proof. Since $X, Y$ are convex polyhedral cones, $X=\{x \mid A \cdot x \leq 0\}$ and $Y=\{y \mid B \cdot y \leq 0\}$ for some matrix $A, B$. Then $X \cap Y$ is defined as $\{z \mid C \cdot z \leq 0\}$ where $C$ is the matrix composed of matrices $A$ and $B$, namely a matrix whose rows are the ones of $A$ and $B$. Thus $X \cap Y$ is a convex polyhedral cone.

A cone $C$ is finitely generated if there exist vectors $x_{1}, \ldots, x_{n}$ such that $C=\left\{\sum_{i} \lambda_{i} \cdot x_{i} \mid\right.$ $\left.\lambda_{i} \geq 0\right\}$. These vectors are called generators of $C$.

Theorem 26 (Farkas-Minkowski-Weyl). A convex cone is polyhedral iff it is finitely generated.

A set of generators of a convex polyhedral cone can be obtained by solving linear equations defining the cone. 
Theorem 27 (Carathéodory's theorem). Let $C$ be a cone generated by vectors of the (finite) set $X$. Then for any vector $x \in C$ there exists a set $Y \subseteq X$ of linearly independent vectors such that $x$ is a vector of the cone generated by the vectors of $Y$.

Lemma 28. The set $\mathcal{H}=\left\{a \cdot \mathbf{i}+F \cdot v \mid a \in \mathbb{Q}^{+} \wedge v \in \mathbb{Q}^{T}\right\} \cap\left(\mathbb{Q}^{+}\right)^{P}$ is a convex polyhedral cone.

Proof. The set $\left\{a \cdot \mathbf{i}+F \cdot v \mid a \in \mathbb{Q}^{+} \wedge v \in \mathbb{Q}^{T}\right\}$ is clearly a convex cone and it is generated by $\mathbf{i}$ and the column vectors of $F$ and $-F$ so it is polyhedral. The intersection of this cone with the cone $\left(\mathbb{Q}^{+}\right)^{P}$ is polyhedral too. 